\title{
FABRICATION AND ANALYSIS OF A PENDULUM PUMP
}

\author{
Rony K Placid", Steffin George $\mathrm{Sam}^{2}$, Amal $\mathbf{R}^{3}$ \\ ${ }^{l}$ Department of Mechanical Engineering, BTCCET, Koothattukulam, Kerala \\ ${ }^{2}$ Department of Mechanical Engineering, BTCCET, Koothattukulam, Kerala \\ ${ }^{3}$ Department of Mechanical Engineering, BTCCET, Koothattukulam, Kerala
}

\begin{abstract}
This paper discusses the importance of a pendulum pump which can be used as a supplementary device for pumping water and is made to replace hand pumps. One important feature of a pump with a pendulum is that the work is alleviated or in simple terms it makes work rather easier when is compared with a traditional hand water pump. It is due to this underlined feature which enables the pendulum pump to be used as an efficient mode in the irrigation of smaller lots, water-wells and can also be used in extinguishing fires even by old people and children. By the use of pendulum based water pumping system we can increase the efficiency of the plant and reduce the effort, cost of production, production time, manpower requirement.
\end{abstract}

Keywords: Work alleviation,energy conservation, dexterity, reciprocating pump, springs,oscillating pendulum

\section{INTRODUCTION}

The ever increasing demand for energy has led to the formation of various advanced resources which produces a certain part of the required energy.One principal consumer of a large amount of energy is our household itself.Large amount of electrical energy is wasted in pumping water,irrigation purposes etc.It is in this context the importance of pendulum pump arises, by the use of which a large amount of energy can be conserved and the conserved energy can be used for various other purposes.

A pump is a device that can be used to raise or transfer fluids. Pumps are selected for processes not only to raise and transfer fluids from one point to another, but also to meet some other criterion. This other criteria may be to obtain a constant flow rate or constant pressure according to the requirement.

The main importance of a pendulum pump is that the initiation energy for starting the process of pumping, swinging of the pendulum, is considerably minimum when compared with the work required to operate hand pumps. Typical hand pumps require sufficiently large effort and an average person can use the pump continuously only for a short time, but the pendulum pump requires only minimum of the effort, because it is only required to oscillate the pendulum and can maintain these oscillation for several hours, without any fatigue. The advantage of this invention compared to present hand pump solutions are: less force to start the pump, less water consumption, and both arms can be used to fetch the water.

\section{PRINCIPAL COMPONENTS}

\subsection{Cycle Frame}

It is the main component of the pump system and is made up of steel. The principal mechanism used for the construction of the pendulum pump is the slider crank mechanism and the frame converts the oscillating movement of the pendulum on one side to the reciprocating motion of the piston to the other side. The cycle frame consists of seven rigid links which converts the pendulum movement into the piston movement. Tension and compression springs are properly fixed to the frame.

\subsection{Reciprocating Pump}

It is a positive displacement pump. It operates on the principle of actual displacement or 'pushing' of liquid by a piston or a plunger that executes a reciprocating motion in a closely fitting cylinder.

\subsection{Springs}

The spring is an elastic object used to store mechanical energy. Here in the pendulum pump both tension and compression springs are used. It is the function of these tension and compression springs to stretch and compress according to the load applied.

\subsection{Tension/Extension Spring}

The spring is made to operate with a tension load, so that the spring stretches freely as the load is applied to it.

\subsection{Compression Spring}

This is made to operate with a compressive load, so that the spring gets shorter as the load is applied to it.

\subsection{Non Return Valves}

A non-return valve or a check valve or one-way valve is a valve that normally allows fluid (liquid or gas) to flow through it in only one direction. 


\subsection{Hose Collar}

A hose collar is used to connect a hose to any other valves or openings. Here it is used to connect the hose to the nonreturn valves at the suction and delivery sides. A hose clamp or is simply called a clip which is used to attach and seal a hose onto a fitting such as a barb or nipple.

\subsection{Nylon Tubes}

Nylon tubes are connected to the delivery and suction ends of the reciprocating pump for the passage of water from the sump and the delivery tank.

\subsection{Weight Hanger}

The weight hanger is used to hold the weights and it is the oscillating part of the system and thus it acts like a pendulum.

\section{CREO PARAMETRIC MODEL}

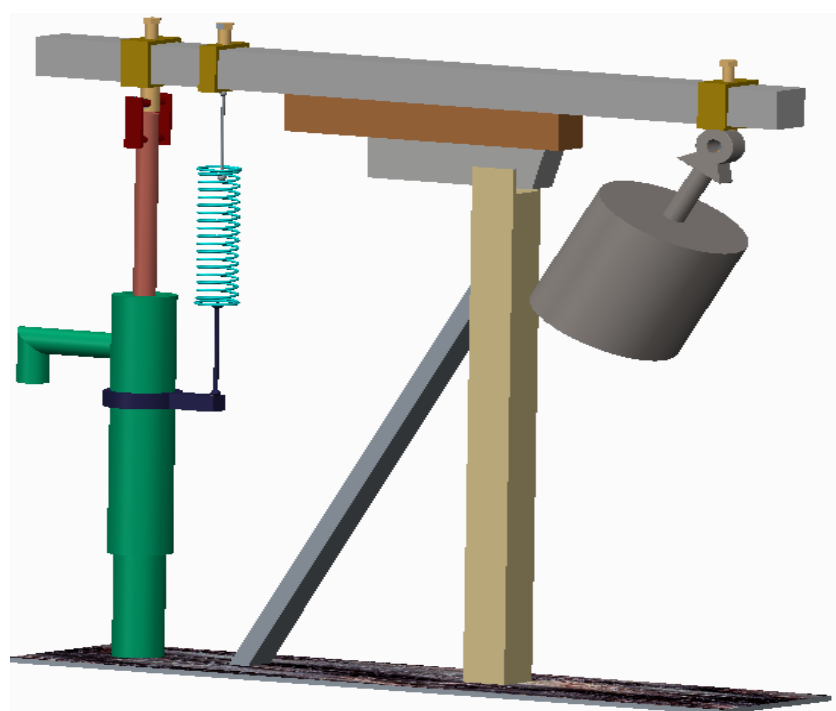

Fig.1 Creo parametric model

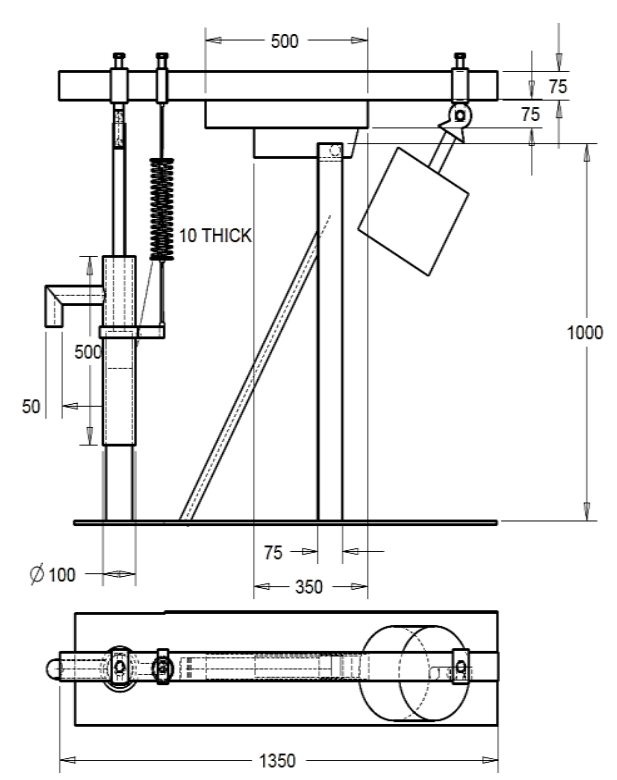

Fig.2 Detailing view

\section{RESULTS AND ANALYSIS}

The various parameters that determine the output discharge of the pendulum pump are analyzed and the results are plotted. Analysis parameters include mass of pendulum, swing angle, length of pendulum.

\subsection{Analysis of Mass of the Pendulum}

Here, the discharge is found out by changing the mass of the pendulum by maintaining the maximum swing angle and maximum length of the pendulum.

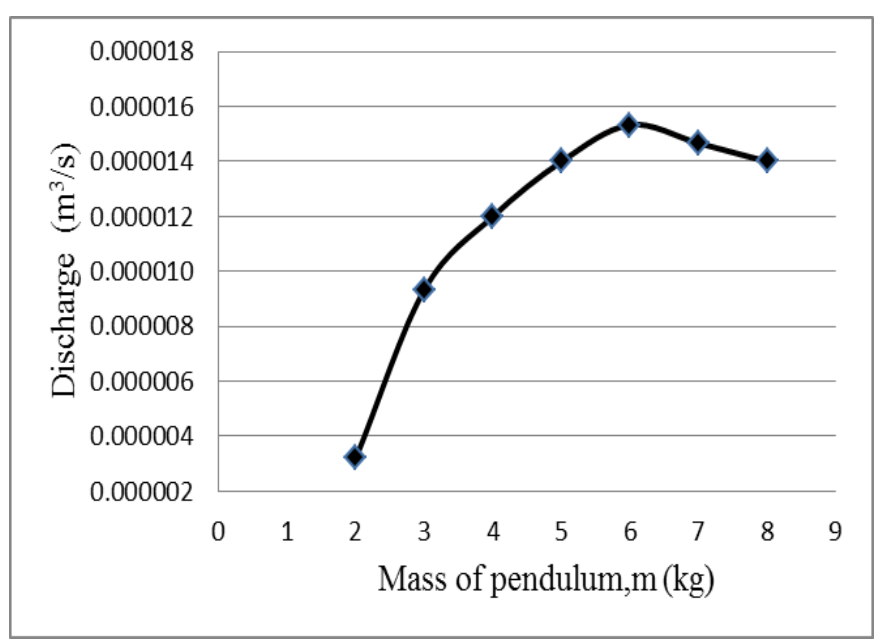

Graph 1 Mass of pendulum, (m) vs Discharge

\subsection{Analysis of Swing Angle of the Pendulum}

Here, the discharge is found out for various swing angles $\left(30^{\circ}, 45^{\circ}\right.$ and $\left.55^{\circ}\right)$ by changing the mass of pendulum without changing the length of the pendulum $(0.55 \mathrm{~m})$.

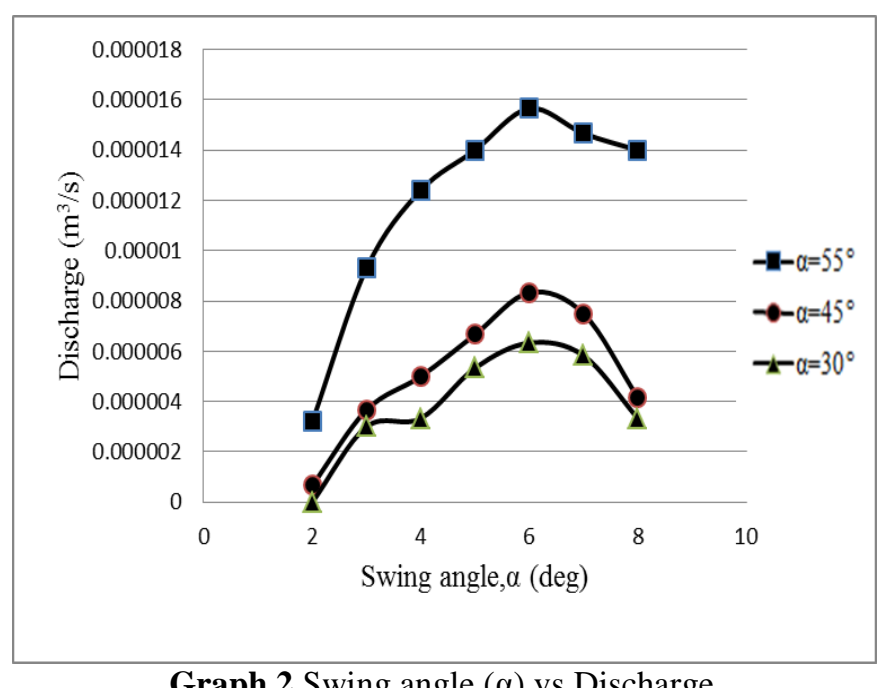

Graph 2 Swing angle $(\alpha)$ vs Discharge

\subsection{Analysis of Length of the Pendulum}

Here, the discharge is found out by varying the length of the pendulum without changing the mass of the pendulum and the swing angle. 


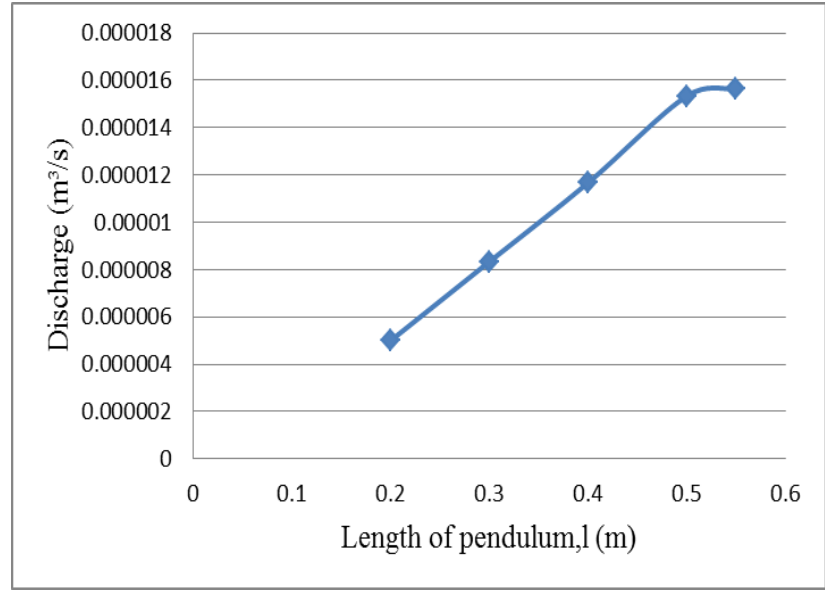

Graph 3 Length of pendulum, (1) vs Discharge

\section{ADVANTAGES \& DISADVANTAGES}

Some of the advantages are

$>\quad$ Uses the minimum of human strength in comparison to present classic hand water pumps.

$>$ In comparison to the hand pumps the energy required to initiate the pumping process is significantly less for the pendulum pump.

$>$ Requires less water consumption.

$>$ Since maintaining the oscillation of the pendulum does not request any special training or dexterity, both parts of the pump can be used to draw fluid from the source.

$>$ Compact size, easy to relocate, less moving parts, hence less maintenance cost

The limitations include

$>$ Less efficiency when compared to other device.

$>$ Air leakage can affect the entire working of the whole unit.

\section{APPLICATIONS}

$>$ Drainage: Used to control the level of water in a protected area.

$>$ Sewage: Used in the collection and treatment of sewage.

$>$ Irrigation: Used to make dry lands agriculturally productive.

$>$ Chemical Industry: Used to transport fluids to and from various sites in the chemical plant.

$>$ Petroleum Industry: Used in every phase of processing of petroleum, its transportation, and separation of the impurities.

$>$ Medical Field: Used to pump fluids in and out of the body.

$>$ Steel Mills: Cooling water in steel mills can be transported using a pendulum pump.

\section{FUTURE SCOPE}

$>$ It can be used where the shortage of power or electricity is a major issue.

$>$ From experimentation it can be concluded that the system is practically feasible.
$>$ Implementing a pendulum pump setup which uses gravitational force only, leads to large amount of energy conservation

$>\quad$ This system is more efficient, considering the minimum efforts required.

\section{REFERENCES}

[1] G. Figliolini, M. Conte and P. Rea, Analysis and Synthesis of Slider Crank Mechanism for automatic machinery, (university of Cassino, Italy).

[2] Chen-Yuan Chung and Yan Shin Shih, Vibration analysis of the flexible connecting Rod with the breathing Crack in a Slider-Crank mechanism,(Jun 19, 2013,Paper No: VIB-12-1306).

[3] Nikhade, G.R.Patil, and Bansal,2009. Two Stage Oscillator Mechanism for operating a reciprocating pump.

[4] Debasish Chatterjee, Amit Patra, Harish K Joglekar, Swing-up and stabilization of a cart-pendulum system under restricted cart track length, 2003 (Department of Electrical Engineering, IIT Kharagpur).

[5] Roshan Kumar, Bhushan Singh, Modelling and simulation of inverted pendulum system using MAT Lab.

[6] P. Melba Mary and N.S.Marimuthu, Minimum Time Swing Up And Stabilization Of Rotary Inverted Pendulum Using Pulse Step Control

[7] Singh Vivekkumar Radhamohan, Mona Subramaniam A, Dr. M.J.Nigam, Fuzzy Swing-Up And Stabilization Of Real Inverted Pendulum Using Single Rulebase,2011( Indian Institute of Technology Roorkee, India). 